\title{
Fuzzy Logic for QoS Control in IMS network
}

\author{
Errais Mohammed \\ Laboratoire d'Informatique \\ LR@II, \\ Faculté des Sciences et \\ Techniques, \\ BP 146 Mohammadia 20650, \\ Maroc
}

\author{
Raouyane Brahim \\ Laboratoire d'Informatique \\ LR@II, \\ Faculté des Sciences et \\ Techniques, \\ BP 146 Mohammadia 20650, \\ Maroc
}

\author{
Bellafkih Mostafa \\ Networks Laboratory, \\ Institut National Des Postes Et \\ Télécommunications, \\ 2, av ALLal EL Fassi - \\ Madinat AL Irfane, Rabat, \\ Morocco
}

\begin{abstract}
The nature of the services provided by IP Multimedia Subsystem (IMS) networks require a maximum quality, this will appear in service provisioning for differentiate between the added value of IMS and other classical case as well as Internet. Toward, the QoS management mechanisms focus primarily on resource reservation in service provisioning, all this specification are introduced by 3GPP standards. Although, all proposed mechanisms haven't any entity overseeing and monitoring services after supply. For that in this work, we propose a new approach QoS monitoring based on fuzzy logic. The technique allows modelling a control system able to identify any $\mathrm{QoS}$ deterioration in real-time, also a rapid resources reconfiguration and restoration to ensure customer satisfaction IMS.
\end{abstract}

\section{General Terms}

Network Management.

\section{Keywords}

IP Multimedia Subsystem (IMS), Fuzzy Logic, Quality of Service (QoS).

\section{INTRODUCTION}

The customer needs in terms of communications technology to multimedia services forcing operators to integrate innovative solutions, for services deployment, supply and assurance. In this sense, the IMS [1] network provides a mechanism for services provisioning regardless network infrastructure as access technology. However, networks diversity and deployed services nature requires the introduction of effective mechanisms for QoS Management and monitoring. For that, the 3GPP specifications offer for IMS networks flexible and dynamic scenarios for configuring resources at services provisioning [2]. The scenarios focus mainly on supply phase but without monitoring mechanisms witch ensure QoS from beginning to end.

Indeed, the multimedia services supply in IMS network requires a continuous QoS monitoring [3] to ensure acceptable reliability and customer satisfaction. Similarly, QoS evaluation is essential to trace the quality provided related to service for comparing thresholds defined in the SLA. And identify any QoS deterioration and configuration management resources accordingly to maintain an acceptable QoS in real-time.
The objective of work is to model a control system based on fuzzy logic [4] for QoS evaluation in real-time in IMS network, considering demands of multimedia services as Video on Demand (VoD) [5]. Indeed, fuzzy logic is a popular technique that provides mechanisms for handling non-linear uncertainties that exist in physical systems. Also, fuzzy logic can supports the natural descriptions of inputs and outputs in terms of language, which avoids the complexity to identify the exact numerical values, to model in each situation. The new approach is based on network performance evaluated via key performance indicators (KPI) which have a considerable impact on audio and video Quality, that for estimate the QoS in real time fuzzy logic-based.

\section{IMS Networks}

The IP Multimedia Subsystem (IMS) is a standardized architecture that enables multimedia services deployment in any access network (wire-line or wireless). The IMS architecture introduces a new distribution to manage networks. It introduces three layers of management control, service and access, each of which focuses on a particular aspect of network management.

The IMS provides a multi-access IP network that allows any broadband interface with the layers of the IMS. Indeed, the IMS network is a set of access networks which inter operate to ensure high service availability and connectivity. The IMS networks offer many advantages to telecom operators including accessibility and connectivity regardless of the access type with rapid integration and services deployment. However, services deployed (streaming) and access networks diversity require the introduction of effective mechanisms for QoS management.

The 3GPP specifications for IMS networks provide a set of adaptable and dynamics scenarios for resource configuration at service provisioning. The scenarios focus primarily on services supply however regardless mechanisms supervision in real time.

We propose a new approach for services supervision based fuzzy logic in IMS Context. The idea consists to model a QoS controller based on resources state. The services monitoring is made using performance indicators that have a direct impact on video streaming.

The design of a controller based on fuzzy logic requires compliance to precise methodology. In particular, the choice of input variables, membership-type functions for these variables and vocabulary used and the intervals to be applied in the phase of fuzzification. It is also important to clarify rules to be applied and membership-type function for the output variable. 


\section{MHETODOLOGIE}

The modelling of a control system based on fuzzy logic consists to identify inputs values, membership-type function and linguistic variables for each input. Before define association rules necessary for inference function, and type linguistic variables of the system output.

The services nature (streaming) supported by IMS requires the control of both streams video and audio, before making a decision on overall QoS. Thus, the proposed control system is composed of three sub control system: one for audio stream, another to control the video and last for estimating the overall QoS-based service output of the first two controllers.

\section{Control system Modelling}

\subsection{Choix of input variables:}

The experiments performed on services nature in IMS networks [6] have been identified, with accurate performance indicators that have considerable impact on the various flows. Performance indicators for the most important in audiovisual streams are as well:

- Delay $(\mathrm{ms})$ : is time required for packets arrival at destination. A critical values of delay causes loss of synchronization between source and destination video.

- $\quad$ Jitter $(\mathrm{ms})$ : variance of delay between packets, which causes loss of synchronization between packets sent.

- Packet Loss (\%): the percentage of packets lost during transmission. this indicator that has not a great impact on audio stream for information amount that is transmitted, but still useful for estimating the QoS in a relevant way.

\subsubsection{Input membership function}

Before presenting functions for each input, it is necessary the identification of linguistic variables chosen for situation model related to each indicator. Indeed, the number of linguistic variables and intervals of each fuzzy subset have a direct impact on performance of control system, in terms of accuracy and time needed to estimate. The subsets used for modelling control system are the same for the three entered for the both streams.

- Low: describe the most optimal subset for all three indicators. Indeed, minimum values show that the estimated QoS is acceptable.

- Medium: describing indicators acceptable.

- High: subset of critical indicators.

The membership functions used are triangular. This choice is explained by nature and variation of key performance indicators (KPI) [7].
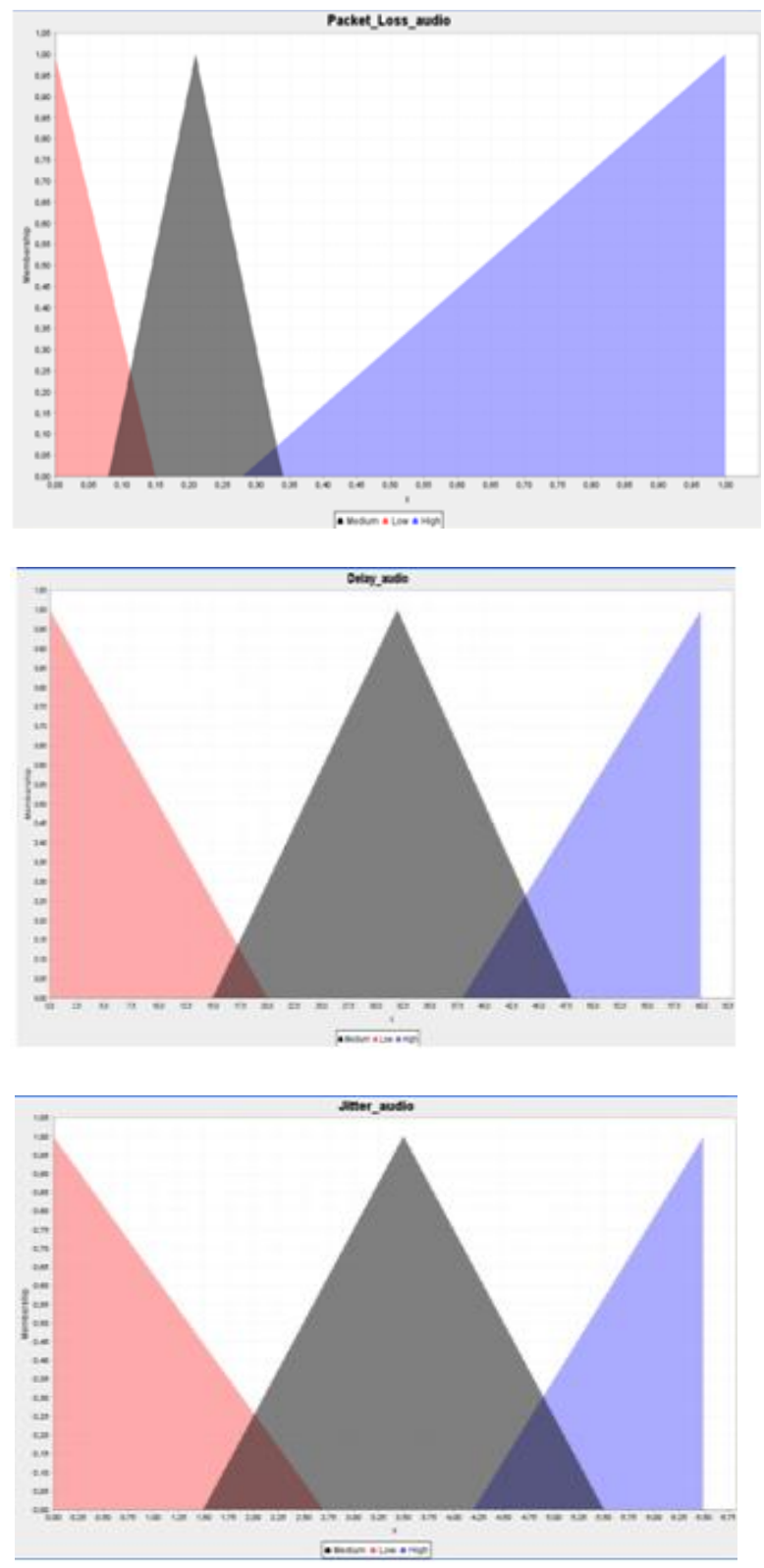

Fig 1: membership functions of inputs for the audio stream

Figure 1 shows the membership function of the three entered Jitter, Delay and Packet Loss for the audio stream. The intersection and thresholds subsets are defined based on experimental study and analysis. 


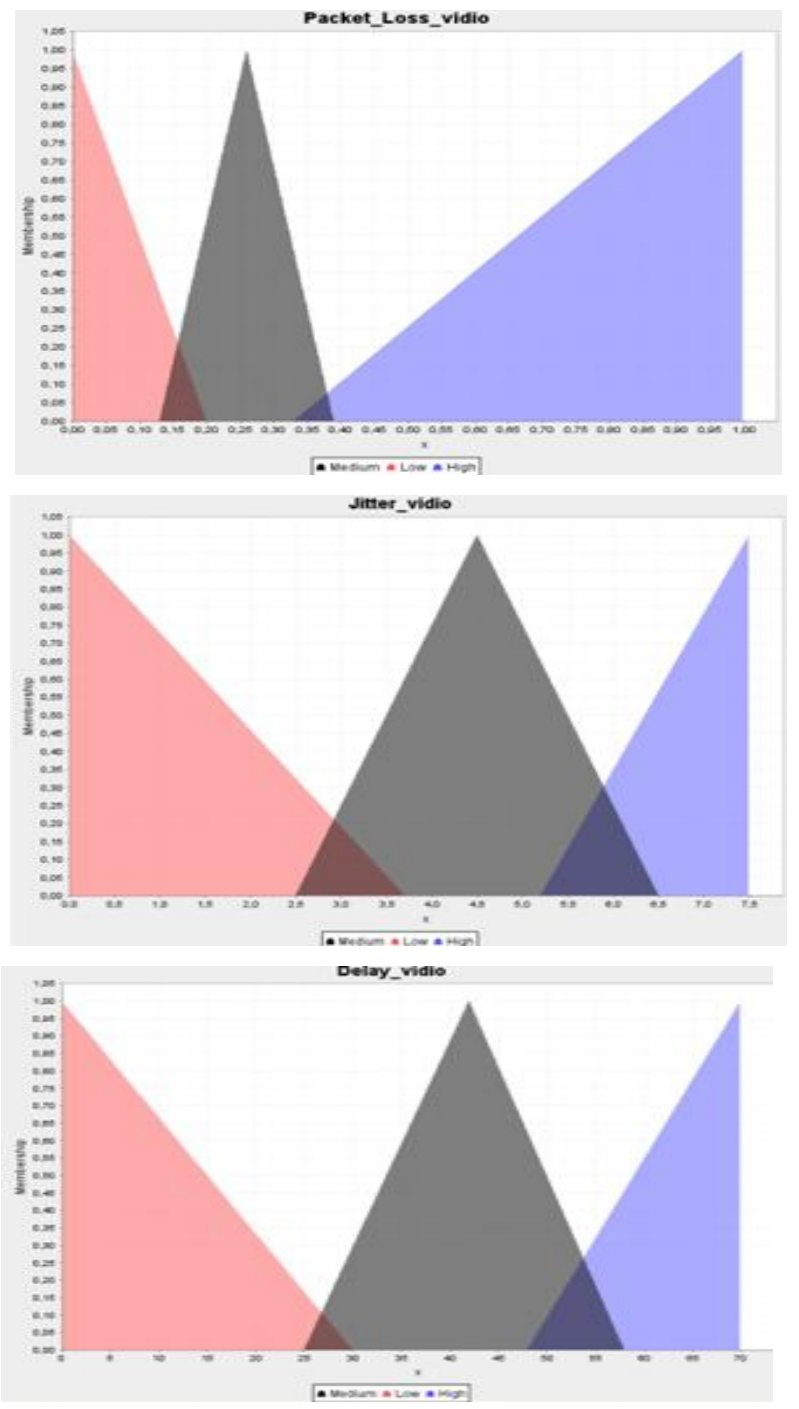

Fig 2: membership functions of inputs for the video stream

The membership-type function is the same for both streams. The difference manifests in terms of thresholds applied, which is explained by sensitivity of the flows and each performance indicator related [6].

\subsubsection{The outputs of QoS controller for audio and video flow}

The QoS estimatation of flows is done through an output for each stream audio-QoS and video-QoS. These two outputs are representing customer satisfaction and audiovisual quality of received video.

The two outputs support three linguistic variables are as follows:

- Excellent: QoS seen as very good and requires no correction, the estimate of satisfaction is over $60 \%$.

- Average: an acceptable QoS but requires verification in case of a major and important customer.
- Poor: QoS is considered critical. Thus, the correction and reconfiguration of resources are needed.
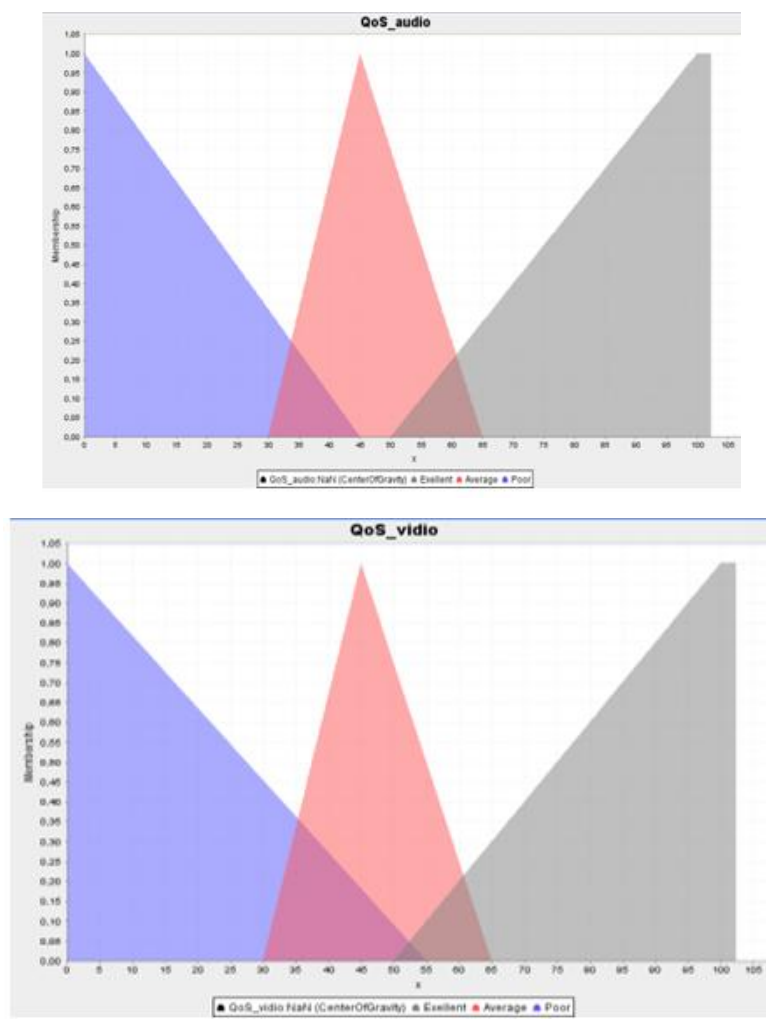

Fig 3: The outputs variables for the controller audio and video

\subsubsection{The overall QoS controller system:}

The objective this approach is introduction of fuzzy logic in the IMS network monitoring to estimate the QoS of streaming services based performance indicators. The approach considers the QoS as a key of customer satisfaction to identify potential problems before surfaced. For this purpose, the QoS estimation in real time is based both audio and video streams. Thus, the QoS control system has indeed three subsystems: audio, video and global for estimate the overall QoS of service (Figure 4).

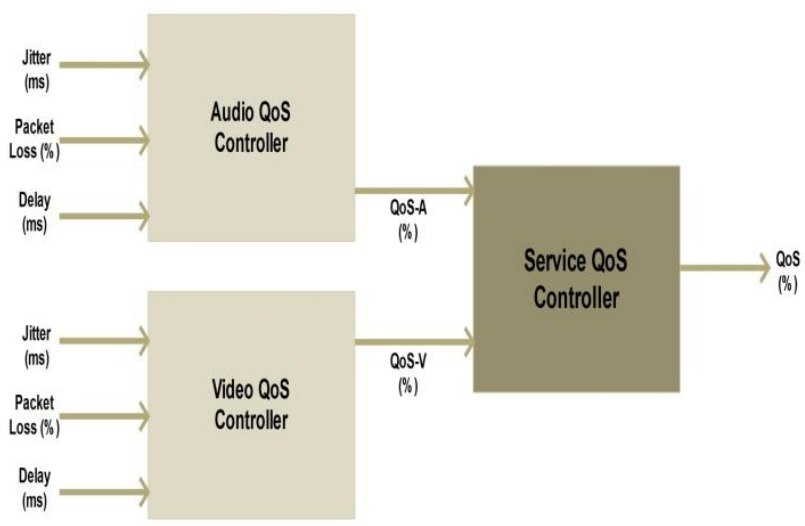

Fig 4: Control System 
The control system QoS is based on the results obtained by both audio and video controllers. The output set a relationship between audio and video to estimate the overall customer satisfaction. The system output is variable QoS, which is the membership-type function is triangular. The linguistic variables used for the output is as follows:

- Critical: a critical QoS that requires correcting the QoS.

- Negotiable: Traded on an acceptable QoS for customers in lower classes.

- Good: an acceptable QoS, including the estimation of satisfaction is over $50 \%$.

\subsection{Architecture System}

The diversity of protocols and entities in IMS network requires the integration of new components to achieve a monitoring system based fuzzy logic. Also, the performance indicators recovery in real time requires innovative solutions in terms of technology used and deployment of monitoring system. The proposed system architecture (Figure 5) consists of several key components to information recovery mandatory for real-time monitoring, including detection of service and its parameters for indicators collection.

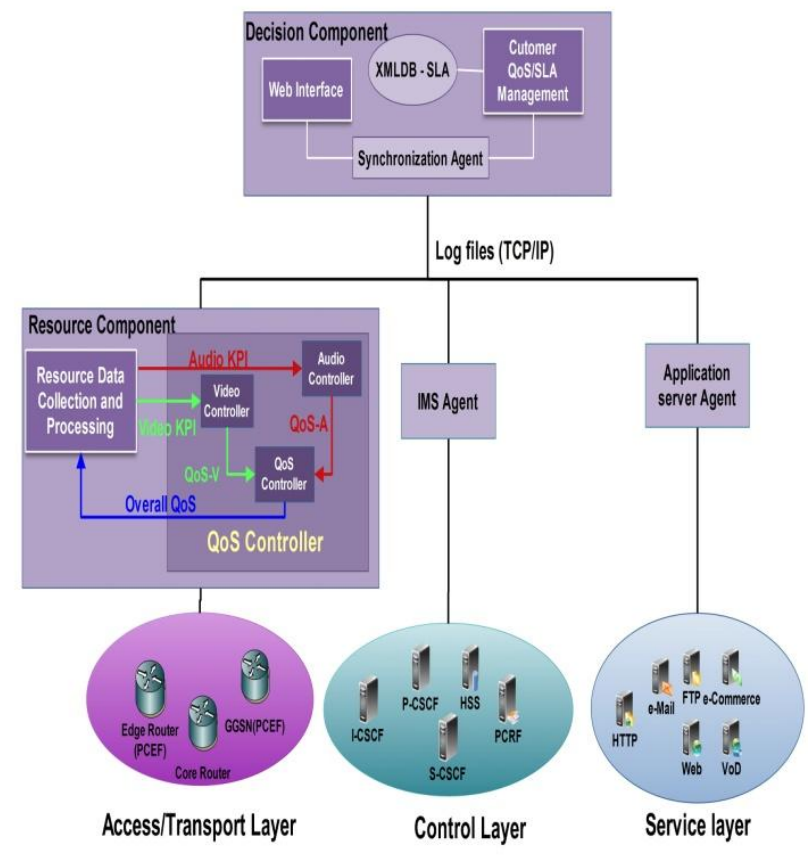

Fig 5: System Architecture

Before presenting the components of monitoring system it is important to introduce a set of deployed agents:

- The IMS agent responsible for detecting service provisioning and the customer's identity through communication with IMS entities in particular $\mathrm{S}$ CSCF and HSS.

- $\quad$ The agent in application server (AS) responsible for collecting service settings such as codec and other parameters.

The QoS system controller is divided in two main components:

- Resource Component that is deployed directly on resources. It has more of the estimation system based on fuzzy logic, a resource data collection [5] module responsible for performance indicators collection in real time.

- Decision Component deployed in server management. It has several entities are as follows:

$\checkmark \quad$ XML-Data Base [8] responsible for safeguarding the SLA [9] storage of registered customers;

$\checkmark \quad$ Web interface for monitoring;

$\checkmark$ A synchronization agent responsible for sync between agents and resource module.

$\checkmark$ Module Customer QoS / SLA Management which ensures compliance with SLA-defined requirements in based results obtained by the control system.

\section{Test and results}

The objective of these experiments is validate the new approach for estimating the QoS-based fuzzy logic, in real cases of services provisioning in IMS networks.

\subsection{Trail architecture}

The test bench (Figure 6) consists of the following:

- A server management that contains the WS-Service and WS-Customer module.

- A router-type core that includes control entities of the IMS solution deployed by OpenIMSCore [10].

- Two types of edge routers that includes WS-Resource module.

- A synchronization server that enables the deployment of WS-Synchronizatrion module and web interface of monitoring.

- A client machine that includes a SIP [11] client supported by IMS (UCTIMSClient).

- An application server VoD media. 


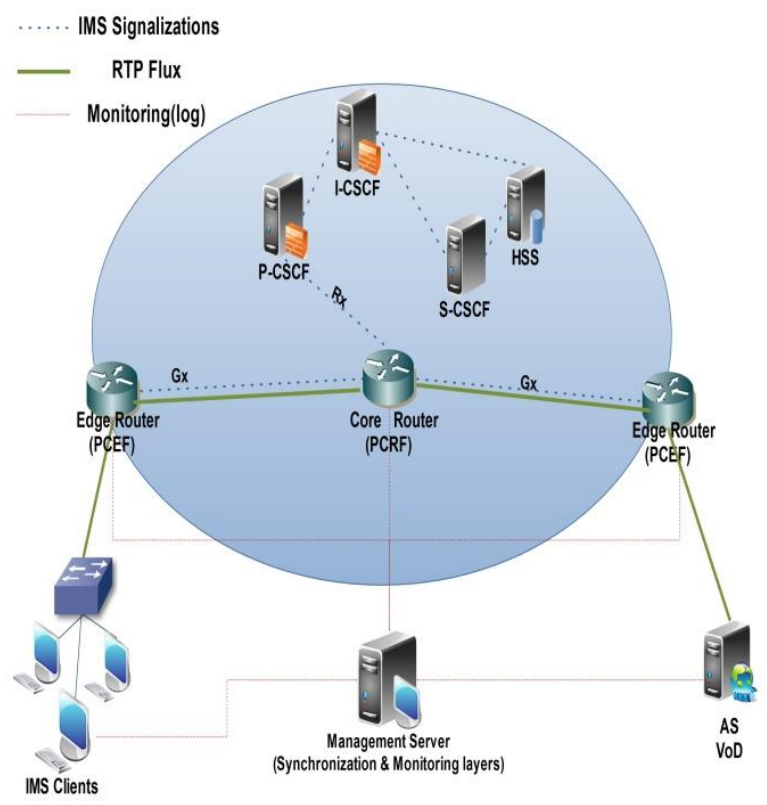

Fig 6: test bench

\subsection{Scenarios}

Initially, the both client Bob and Alice, with type is platinum and Gold respectively, registering in the IMS network before requesting VoD service. Then, we proceed to overload network by generating traffic through Iperf tool [12]. Subsequently, the number of flows is doubled. In all three cases, the estimation of QoS is achieved via the platform.

\subsection{Results}

In the first case, the value of output variable of control system is 90\%. A value that reflects the QoS received (Figure 7). This is due to absence of competing flows in network and thus minimizing queuing at routers in resource.

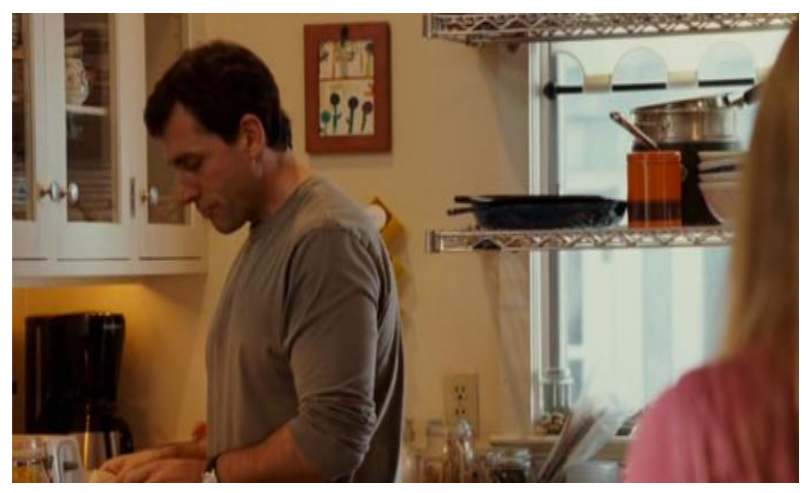

Fig 7: The captured image in the first case

In the second case, flows competitor overhead network. As a result, the appearance of static pixels in received video (Figure
8) as well as voice quality drops significantly. This deterioration affects the output variable value that expects $57 \%$.

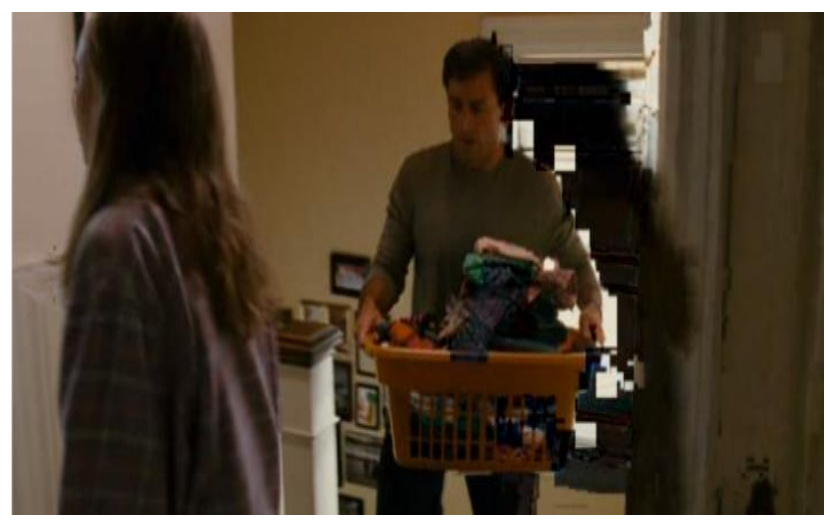

Fig 8: The captured image in the second case.

In the third case, QoS become critical (Figure 9), which is explained by the presence of several users and competing flows in terms of resource in the network. The QoS value estimated by system does not exceed $27.45 \%$.

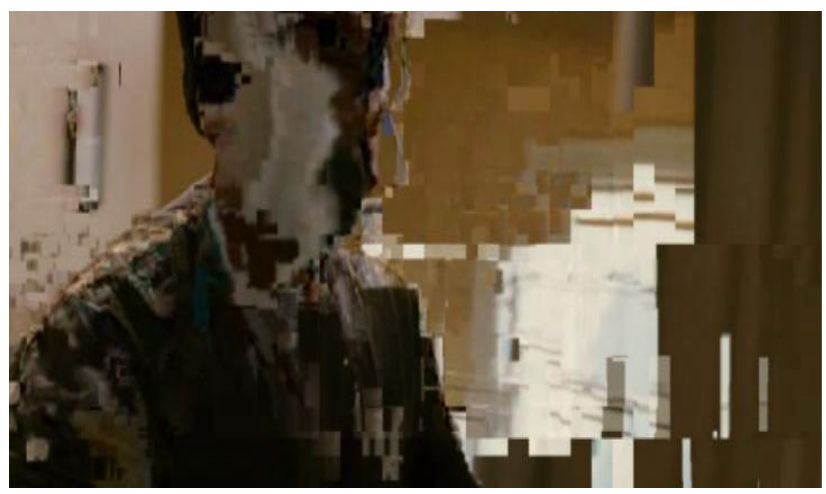

Fig 9: The captured image in the third case.

\subsection{Discussion:}

The control system of QoS-based fuzzy logic allows estimating QoS successfully and in real time. However, it is important to assess the costs of such an approach in terms of resources and time for degradation detection. The response time of control system is presented as the most important decisive factor of evaluation. 


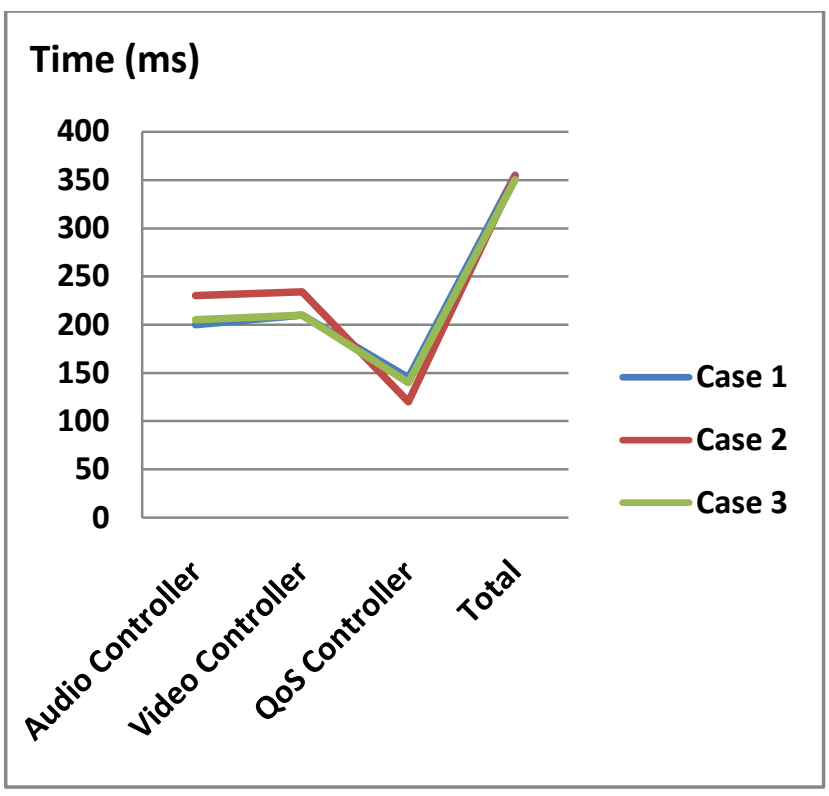

Fig 10: The execution time of the QoS control system

The execution time is composite, it depends on the time required for the three controller (audio, video and QoS), and the number of input variables defined for each controller. Indeed, increasing numbers of input variables can change precision in the estimate result as well as execution time. Hence, we choice to not introduce a supplementary input like bandwidth. Similarly the threads use in implementation of controller has significantly minimized the time of estimation.

The second criterion is the detection time of QoS degradation, with and without the use of the control system (Figure 11).

\section{Time (ms)}

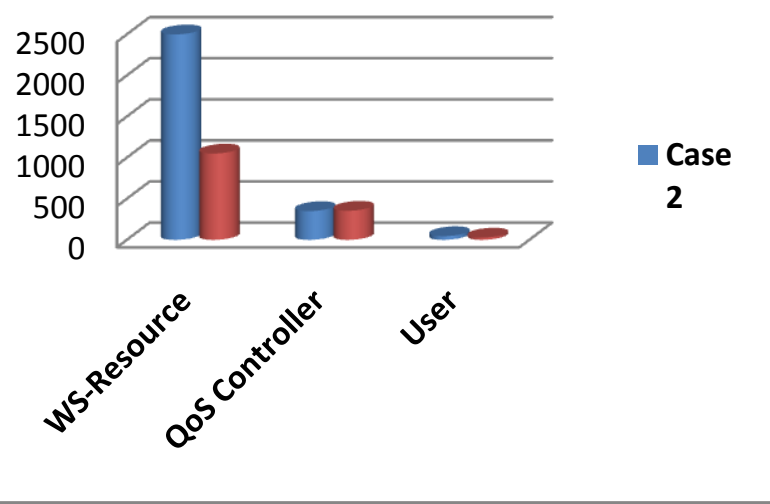

Fig 11: The detection time of the degradation of QoS.

Figure 11 describes the detection time of QoS deterioration in both cases 2 and 3 with control system, but also when applying thresholds in RDCP (Resource Data collection \& Processing)
[13] without activation of the control system. Time identification of degradation via the static thresholds is very important $(2500 \mathrm{~ms})$, reflecting the need to wait until the thresholds are exceeded. One approach considered limited to the large time difference between the defection and the client system.

The control system integration fuzzy logic-based has reduced significantly the detection time $(350 \mathrm{~ms})$, which will correct the deterioration in optimum time.

\section{CONCLUSION}

The new approach integration for QoS estimation in real time based on fuzzy logic was used to evaluate IMS networks in terms of efficiency and reliability. The approach aim is the introduction of effective mechanisms for monitoring real-time services in IMS networks. The objectives achieved through the ability to identify potential user satisfaction in terms of QoSbased performance indicators collected in real time. The choice of input and output variables has a significant impact on the effectiveness and costs required to deploy the control system.

Thus, the use of three entered for each audio and video controller for combining both for overall QoS has achieved the best performance, in terms of detection time and stability regardless of the number of concurrent streams or network status.

In order to achieve an autonomous system based eTOM processes and fuzzy logic to the identification and real-time correction of any deterioration in QoS. We propose in our future work to match control system based on fuzzy logic to the scenarios for correcting the eTOM in the context of IMS.

\section{REFERENCES}

[1] Poikselka, M. and Georg M. (2009) The IMS: IP Multimedia Concepts and Services, John Wiley \& Sons Inc. Chichester, England.

[2] BELLAFKIH Mostafa, RAOUYANE Brahim, ERRAIS Mohammed, RAMDANI Mohammed "QoS Management in IMS: Diameter-DiffServ". NGNS'10: International Conference on Next Generation Networks \& Services, 0406 juin 2010, Rabat, Maroc.

[3] ERRAIS, M; Rouayane, B; Bellafkih, M; Ramdani, M; "Enhanced Telecom Operation Management For IMS Networks" ; International Journal Of Next-Generation Networks Vol. 3, No. 2; June 2011.

[4] Timothy J.Ross (2004) Fuzzy Logic with Engineering Applications, John Wiley \& Sons Inc. Chichester, England.

[5] Leghroudi, D; ERRAIS, M; Moumkine, N;Bellafkih, M; Ramdani, M; "eTOM Process to Manitor and Corelat IPTV QoS in IMS" ; International Journal Of Next-Generation Networks Vol. 3, No. 2; June 2011.

[6] Bellafkih, M.; Raouyane, B.; Errais, M.; Ramdani, M. , "MOS evaluation for VoD service in an IMS network," I/V 
Communications and Mobile Network (ISVC), 2010 5th International Symposium on , vol., no., pp.1-4, Sept. 30 2010-Oct. 22010.

[7] SERIES M: Telecommunications management network Enhanced Telecom Operations Map (eTOM) Representative process flows, ITU-T Recommendation M.3050.

[8] Extensible Markup Language (XML) 1.0. Tim Bray et al. World Wide Web Consortium. http://www.w3.org/TR/2009/REC-xmlbase 20090128/

[9] Errais, M.; Bellafkih, M.; Raouyane, B.; Ramdani, M, "Distributed network Monitoring for IMS Network", The 2nd International Conference on Multimedia Computing and Systems (ICMCS'11), 7-9 April 2011, Ouarzazate, Morocco.
[10] OpenIMScore - Open source implementation of IMS Call Session Control Functions and Home Subscriber Service (HSS) -http://www.openimscore.org/

[11] SIP: Session Initiation Protocol, June 2002, RFC 3261

[12] IPERF - http://iperf.fr/

[13] Raouyane Brahim, Bellafkih Mostafa, Errais Mohamed, Ramdani Mohamed, "IMS management and monitoring with eTOM framework and composite web service" International Journal of Multimedia Intelligence and Security, Vol. 2, No. 2, pp.172-185. To appear in the end 2011. 